\title{
An NMR Study of the Effect of Polymerization Methods on Segmented Sequence Distributions of Poly(butylene terephthalate)/Poly(tetramethylene glycol) Block Copolymers
}

\author{
Bumchan MIN ${ }^{\dagger}$ and Eunjung BANG* \\ Analytical Science Center, Samyang Central Research Institute, \\ 63-2 Hwaam-Dong, Yusung-Gu, Taejeon 305-348, Korea \\ * Korea Basic Science Institute, 5-126-16 Anam-Dong, \\ Sungbuk-Gu, Seoul 136-701, Korea
}

(Received June 1, 1998)

\begin{abstract}
Sequence distributions and microstructures of block copolymers of poly(butylene terephthalate) and poly(tetramethylene glycol) (PBT/PTMG) were investigated by NMR spectroscopy. The two block copolymers that had same chemical compositions, but were expected to have different sequence distributions were synthesized by two different polymerization methods differing in feeding time of PTMG (sample I, one step feeding; sample 2, two step feeding). To figure out the small differences in microstructures of the two copolymers using NMR, the choice of solvents, the NMR experimental conditions, the ${ }^{1} \mathrm{H}$ and ${ }^{13} \mathrm{C}$ peak assignments and the methods of extracting sequence distributions were closely reexamined. Using phenol- $d_{6} /$ tetrachloroethane (TCE) as NMR solvent, various kinds of controlled 1D and 2D experiments were carried out for peak assignment and close quantification of the peaks. Next, the various parameters relating to the sequence distributions were calculated and those were compared for two samples. As the results, the sample that was made with one step feeding of PTMG was found to have nearly the same sequence distributions as expected by feed ratio. Though there were slight variations in microstructures of two samples, detailed statistical treatment (error analysis) of 10 independent data showed that there were not significant differences in sequence distributions. In the end of this report, the differences in the status of terminal groups of two samples were discussed by manifest ${ }^{1} \mathrm{H}$ signals.

KEY WORDS Poly(butylene terephthalate)/ Poly(tetramethylene glycol) / Triad Sequences / Sequence Distributions / Microstructures / Nuclear Magnetic Resonance /
\end{abstract}

Block copolymers of poly(butylene terephthalate) and poly(tetramethylene glycol) (PBT/PTMG) are of great importance in the industry of thermoplastic elastomers. Because of their excellent mechanical properties and inexpensive production cost, they are widely used as fibers and engineering plastics. As in the case of other thermoplastic elastomers, PBT/PTMG consists of hard and soft segments. Generally, it is well known that, in the morphological point of view, hard segments tend to form semicrystalline domains whereas soft segments play as the matrix of rubbery amorphous regions. ${ }^{1-3}$ Then, the size and distribution of each phase (crystalline and amorphous) can directly affect the mechanical properties of the elastomers.

Among the various factors that determine crystalline phase, the effect of segment lengths and microstructures were widely debated. ${ }^{4-7}$ Of course, a major factor controlling segment length is the composition of the polymer. Because there is low probability of two or more adjacent PTMG occurring (the segment length of soft segment is nearly equal to unit) due to long length of PTMG $\left(M_{n}\right.$ is $\left.1000-2000\right)$, the average hard segment length is mainly determined by the hard segment contents (HSC). However, some authors report that there is considerable amount of long sequences of soft segment when the HSC becomes sufficiently low, then, consequently, an average soft segment length becomes greater than unit. ${ }^{8}$ At this time, the major factor controlling the segment length is also considered to be the HSC. However, one can also expect that there are some variations in the microstructures (such as segment lengths,

\footnotetext{
$\dagger$ To whom correspondence should be addressed.
}

sequence distributions, etc.) of the copolymer owing to the preparation and processing conditions. A recent report associated with the transesterification and change in the microstructures of the blends of poly(ethylene terephthalate) (PET)/ $\mathrm{PBT}^{9}$ implies that there are also some possibilities of variations in sequence distributions including triads sequences (Figure 1) in the case of PBT/ PTMGs containing same chemical constituents.

The present study mainly is aimed at a close comparison of the microstructures of two polymers that were prepared by two different polymerization methods. The main tools of the investigation were various NMR methods that were used by other authors. ${ }^{3,8-10}$ Recently Jacques et al. ${ }^{9}$ reported that the ${ }^{13} \mathrm{C}$ NMR method has some limitations when utilized for the precise quantification of segment distributions. In this study, to overcome the limitations, all procedures of measurements and data analysis were closely reinvestigated including choice of solvents, NMR experimental conditions, ${ }^{1} \mathrm{H}$ and ${ }^{13} \mathrm{C}$ peak assignments and methods of extracting sequence distributions. Detailed error analyses were undergone for several experiments to figure out the significance of the difference between two samples.

\section{EXPERIMENTAL}

\section{Samples}

The methods of synthesizing the PBT/PTMG block copolymers have been reported elsewhere by several other authors. ${ }^{1-13}$ Our two samples (sample 1 and 2) followed similar procedures. Based on those methods, we tried to impose the different polymerization conditions to affect the microstructures of the polymers. In a 
little detail, the two samples were prepared as follows. Sample 1 was prepared by two-stages melt polymerization in a lab scale polymerization reactor. In first stage, the commercial grade dimethyl terephthalate (DMT), 1,4-butanediol (BD), and PTMG with number average molecular weight 2000 were mixed, heated up to $145^{\circ} \mathrm{C}$ with stirring under nitrogen atmosphere. This ester interchange $(\mathrm{EI})$ reactions were performed for $3 \mathrm{~h}$ with $t$-butyl titanate as catalyst. Then, at the beginning of second stage, the reactor was sealed and evacuated below 1.0 Torr, and simultaneously heated up to $250^{\circ} \mathrm{C}$ to conduct polycondensation (PC) reaction. After $2 \mathrm{~h}$ polycondensation reaction, the polymer was poured into cooling water and cut into chips to use for further studies. The relative amounts of starting materials were adjusted for $\mathrm{HSC}$ of the polymer to be $33.3 \%$. To prepare sample 2, under the same polymerization process, the feeding of PTMG was regulated as follows; one fifth of total PTMG was fed at the beginning of EI step and four fifths was at the beginning of PC step. The prepared polymer was used without further purification. We will refer the methods of polymerization as follows; method 1 for sample 1 and method 2 for sample 2 .

\section{Molecular Weight Distributions and Melting Tempera- tures}

The molecular weight distribution of each polymer was measured on the Waters 150-C ALC/GPC using three serial $\mu$-Styragel HT3, HT4, and HT5 columns. $m$-Cresol was employed as elution solvent at the temperature of $100^{\circ} \mathrm{C}$. After elution, the molecular weight was calibrated with polystyrene standard. The melting point of the polymers was measured by Perkin Elmer DSC-4 under the heating ratio of $20^{\circ} \mathrm{Cmin}^{-1}$ after calibrating using indium. To remove previous thermal history, the polymer was heated up to $200^{\circ} \mathrm{C}$ from $25^{\circ} \mathrm{C}$, cooled down to $25^{\circ} \mathrm{C}$, and reheated up to $300^{\circ} \mathrm{C}$ for data acquisition.

\section{Solvent Test}

For the purpose of finding good solvents to go on further NMR studies, we tried four solvent systems; TFA- $d, \mathrm{CDCl}_{3}$, TFA- $d / \mathrm{CDCl}_{3}$ (1:1 mixture by volume), phenol- $d_{6} /$ tetrachloroethane (TCE) (1:1 mixture by volume). To make $10 \%(\mathrm{w} / \mathrm{v})$ solution, appropriate amount of the polymer was put into the NMR solvent and allowed to rest in room temperature for more than one night. First, the states of the resulting solutions were inspected by naked eyes, and then the exact chemical compositions were detected through following ${ }^{1} \mathrm{H}$ NMR measurements.

\section{NMR Experiments}

Bruker AMX-500 NMR spectrometer equipped with temperature controller was utilized for all NMR experiments. All NMR experiments were made on the solution in $5 \mathrm{~mm}$ NMR tube using above four solvents of which concentration was adjusted to be $10 \%(\mathrm{w} / \mathrm{v})$. For good resolution of the spectra, temperature was maintained at $80^{\circ} \mathrm{C}$ during the experiments for the samples in phenol- $d_{6} /$ TCE. For the samples made by TFA- $d, \mathrm{CDCl}_{3}$, and TFA- $d / \mathrm{CDCl}_{3}$ the temperature was maintained at $30^{\circ} \mathrm{C}$ instead of high temperature because of low boiling point of the solvents. ${ }^{1} \mathrm{H}$ NMR
Table I. Chemical compositions, molecular weights, and melting temperatures of two samples

\begin{tabular}{cccccc}
\hline Samples $^{\mathrm{a}}$ & $\mathrm{HSC} / \% \mathrm{o}$ & $M_{n}$ & $M_{w}$ & MWD & $T_{\mathrm{m}} /{ }^{\circ} \mathrm{C}$ \\
\hline 1 & 33.30 & 172758 & 243329 & 1.41 & 185.7 \\
2 & 33.30 & 152741 & 227531 & 1.49 & 185.0 \\
\hline
\end{tabular}

${ }^{a}$ Samples 1 and 2 are prepared respectively from the polymerization methods 1 and 2 in the text. ${ }^{b}$ Hard segment contents (from feed ratio).

experiment was performed using $30^{\circ}$ pulse with long relaxation delay for quantitative analysis. In detail, ${ }^{1} \mathrm{H}$ NMR spectra were acquired through following experimental conditions; rf pulse width of $3 \mu \mathrm{s}$, acquisition time of $2.6 \mathrm{~s}$, relaxation delay of $5 \mathrm{~s}$ and number of scan of 16. Before main acquisition of ${ }^{13} \mathrm{C}$ NMR spectra, carbon relaxation times were estimated via null point method to determine relaxation delay for following acquisition. Then ${ }^{13} \mathrm{C}$ NMR spectra were obtained under the condition of suppression of NOE during relaxation delay while proton noise decoupling during the acquisition (inverse gated decoupling) using rf pulse width of $8.0 \mu \mathrm{s}$, relaxation delay of $14 \mathrm{~s}$ and number of scan of 10000 to 20000. We prepared five NMR samples for each sample. Then, for all NMR samples, ten sets of ${ }^{1} \mathrm{H}$ and ${ }^{13} \mathrm{C}$ NMR spectra were repeatedly obtained using above conditions. To perform clear assignment of small peaks of aliphatic regions in ${ }^{1} \mathrm{H}$ NMR spectra, ${ }^{1} \mathrm{H}-{ }^{1} \mathrm{H}$ COSY experiments were made by making use of standard pulse sequence of Bruker spectrometer. Good quality of spectra could be obtained after processing using qsinebell function. For full assignment of ${ }^{13} \mathrm{C}$ NMR peaks in aliphatic regions, ${ }^{1} \mathrm{H}-{ }^{13} \mathrm{C}$ COSY (HETCOR) were also obtained to see direct heteronuclear coupling, especially between the small peaks of ${ }^{13} \mathrm{C}$ and ${ }^{1} \mathrm{H}$ NMR in aliphatic regions. For exact peak assignment of the fine structures of ${ }^{1} \mathrm{H}$ and ${ }^{13} \mathrm{C}$ peaks of phenyl and carbonyl regions, the experiments revealing heteronuclear long range couplings were needed. For that purpose, we used indirect experiment (HMBC) because of low sensitivity of long range couplings in the polymers. To obtain 2D spectra with good digital resolution, very narrow ranges of spectral widths were used for both axes to result in FID resolution of $0.4 \mathrm{~Hz}$ for proton and $4 \mathrm{~Hz}$ for carbon.

\section{RESULTS AND DISCUSSION}

\section{Molecular Weight Distributions and Melting Tempera- tures}

The measured molecular weights and melting temperatures of two samples are presented in Table I. Both of the polymers are shown to have number average molecular weights larger than 150000. In the point of repeating units of our samples, it corresponds to about 50 units of PTMG and 250 units of BD considering the molecular weights and fed quantities of the starting materials. Therefore we took these facts into accounts in further explanation of our NMR data, especially the effects of terminal group of the finite length of the polymers. From the table, we can see the molecular weight of the sample 1 is slightly larger than that of sample 2. In general, the molecular weights of the 
polyester copolymers can be controlled by the duration of polymerization. For sample 2, due to the lagging of the time of feeding major parts of PTMG in its preparation, total reaction time of PTMG of sample 2 may be considered shorter than that of sample 1 . Therefore it may play as the limiting factor in growing of molecular weight of sample 2 . However, we think that the slight difference in molecular weight between two samples does not affect significantly the physicochemical properties of the samples. The nearly identical melting temperatures for two samples support above thinking.

\section{Stability and Solubility of the Samples against Selected Solvents}

As previously described, we tested four solvents to find good ones for NMR study. Generally, because the polymers having different chemical compositions have also different solubility against one solvent, the good solvent that dissolves all the samples is essential for comparison of different samples. For the purpose of NMR analysis of many kinds of terephthalate polyesters, TFA-d or its cosolvent were commonly used. For PBT/PTMGs and other polyether esters (PEE), $\mathrm{CDCl}_{3}$ was also used for the polymer having low contents of hard segment. ${ }^{8,14}$ Phenol/TCE is typical solvent in measurement of molecular weight and intrinsic viscosity of the terephthalate polyester. ${ }^{13,15}$ Apparently, our samples seemed to be fully dissolved in above four tested solvents. However, from examination of proton spectra, we found that degradation of the sample occurred when solvents containing TFA were employed. Though, we will not present the experimental results in detail, the main chemical reaction in the degradation seemed to be severance of ether linkage in PTMG to form butanediol (degradation of about $5 \mathrm{~mol} \%$ within 1 day). Therefore we did not perform further study for those solvents. In contrast, two samples in $\mathrm{CDCl}_{3}$ and phenol- $d_{6} /$ TCE were remained with no chemical change for several months. For each sample, the chemical compositions of two solutions (in $\mathrm{CDCl}_{3}$, phenol$\left.d_{6} / \mathrm{TCE}\right)$ were compared each other. It can be done mainly by the comparison of the NMR spectra. Through the simple comparison of the two ${ }^{1} \mathrm{H}$ NMR spectra from two solutions, we could find that the ratios of integration of the proton signals of soft segments to hard ones did not accord with each other. If we admit that the solvating power of phenol/TCE is stronger than that of $\mathrm{CHCl}_{3}$ for our samples, the result means that some parts of hard segments of the sample are not dissolved in $\mathrm{CDCl}_{3}$. In following sections, the comparison of exact chemical compositions of the two samples will be presented through calculation using ${ }^{1} \mathrm{H}$ and ${ }^{13} \mathrm{C}$ NMR data.

\section{${ }^{1} \mathrm{H}$ and ${ }^{13} \mathrm{C}$ NMR Peak Assignments}

As previously described, because only phenol/TCE was appropriate to perform this study, we will mainly present the results of NMR experiments from that solvent. Many works relevant to the compositional and micro structural characterizations of polyester copolymers were performed through interpretation of ${ }^{1} \mathrm{H}$ and ${ }^{13} \mathrm{C}$ NMR spectra, and those were based on the peak assignments of the NMR spectra. ${ }^{8,12,14,15}$ Those were mainly related to the simple peak assignments of major peaks in aliphatic

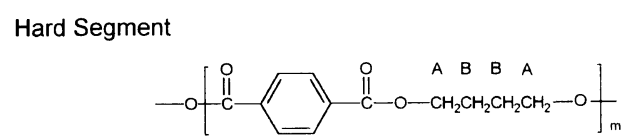

Soft Segment

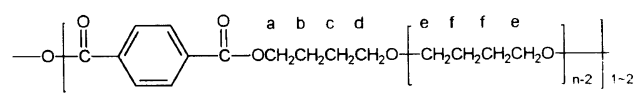

Four Possible Triads

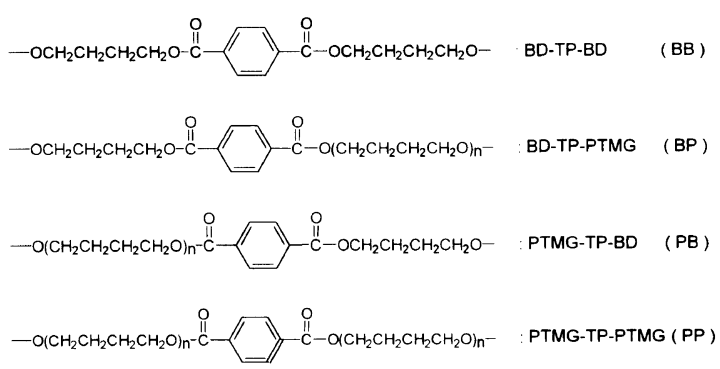

Figure 1. The schematic representation of PBT/PTMG and four possible triad sequences.

and aromatic regions of the spectra. When we closely examine the aliphatic region of the high resolution ${ }^{1} \mathrm{H}$ and ${ }^{13} \mathrm{C}$ NMR spectra of PBT/PTMG, we can find some small peaks beside the main peaks. In addition, close examination of aromatic and carbonyl regions of ${ }^{1} \mathrm{H}$ and ${ }^{13} \mathrm{C}$ NMR spectra make us find that the peaks are minutely split into three lines in phenol- $d_{6} /$ TCE (Figure 2 ). We found that these split peaks had some relationship with triads sequences of PBT/PTMG and all these peaks could be used simultaneously to extract the information relevant to the microstructures of the polymers. In their paper, ${ }^{8}$ Higashiyama et al. assigned the four peaks in the aromatic region of ${ }^{13} \mathrm{C}$ NMR spectra obtained from $\mathrm{CDCl}_{3}$ solution. They suggested that the four peaks were attributed from different environments of aromatic quaternary carbons in four possible triads; BD-TP-BD, BD-TP-PTMG (BD site), PTMG-TP-BD (PTMG site), PTMG-TP-PTMG (Figure 1). From the integration of four peaks of quaternary carbon, they could get the information relevant to the probability of four possible triads, though we found that there were some mistakes in the peak assignments and calculations of sequence length in the paper.

In the case of our samples, when we used $\mathrm{CDCl}_{3}$, we could get similar NMR patterns as those in the previous paper. However, from the spectra using phenol- $d_{6} / \mathrm{TCE}$, only three lines were found instead of four lines for those atoms (the peaks among $133.5-113.9 \mathrm{ppm}$ in Figure 2). A quick comparison of the relative intensities of the lines in the spectra from two solvents made us think that PTMG-TP-PTMG peak that was clearly visible in the spectra from $\mathrm{CDCl}_{3}$ was overlapped into the middle peak associated with BD-TP-BD in the spectra from phenol- $d_{6} /$ TCE. Of course, it is impossible to extract full information relevant to the probabilities of four possible triads from only three lines of quaternary carbons as Higashiyama did. Therefore it was necessary to find other methods to go on further study using 


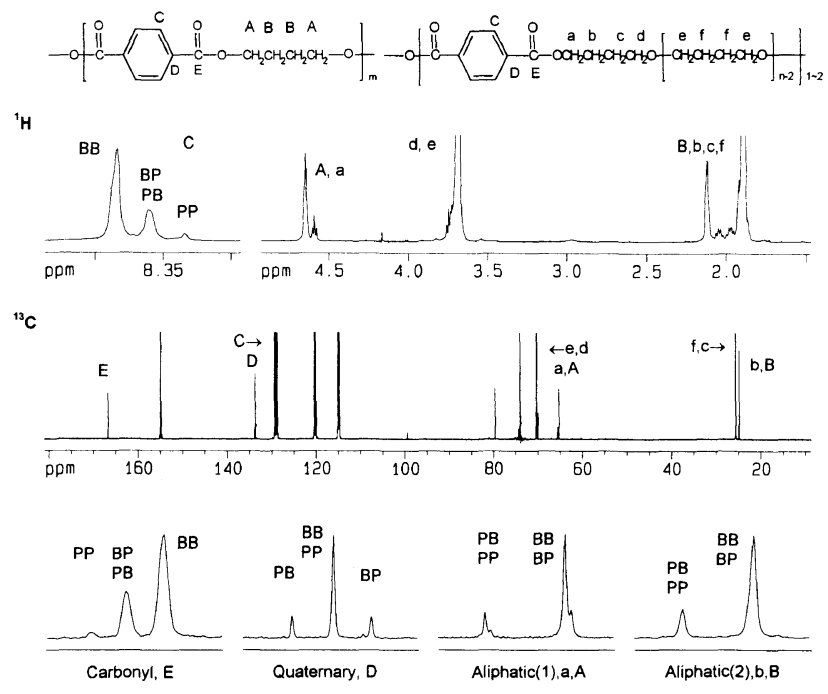

Figure 2. ${ }^{1} \mathrm{H}$ and ${ }^{13} \mathrm{C}$ NMR spectra representing the splitting patterns of selected peaks according to the chemical structures and microstructures of PBT/PTMG.

phenol- $d_{6} /$ TCE. We tried to overcome the difficulty through finding more peaks including the information relevant to the microstructures of the polymers. First, we had attention to the carbonyl peaks and could see that the three lines of carbonyl carbons also contain some information relevant to the microstructures of the polymer. Actually, in the spectra from phenol- $d_{6} / \mathrm{TCE}$, we could find that the sum of integration values of two side peaks of aromatic quaternary carbons was very close to the integration of middle peak of carbonyl carbon. Moreover, the sum of integration of left and right peaks of carbonyl carbon was found to be close to the integration value of middle peak of aromatic quaternary carbon. Through above results we could assign six peaks of aromatic quaternary and carbonyl carbons as follows; for quaternary carbon, left peak to PTMG-TPBD (PTMG site), middle peak to BD-TP-BD and PTMG-TP-PTMG, right peak to BD-TP-PTMG (BD site); for carbonyl carbon, left peak to PTMG-TPPTMG, middle peak to BD-TP-PTMG (BD site) and PTMG-TP-BD (PTMG site), right peak to BD-TP-BD.

In addition to above six lines in ${ }^{13} \mathrm{C}$ NMR spectrum, we could find that the peaks corresponding to the aromatic proton in ${ }^{1} \mathrm{H}$ spectra that were also split into three lines in phenol- $d_{6} /$ TCE have some relationship with triad structures of the polymer. When the peaks of aromatic protons and those of carbonyl carbons are compared, it can be found that the patterns of the peaks are very similar except inversion of the order of peak intensities with each other along to the chemical shift. The fact makes us able to suggest that the peaks of phenyl proton also have some information relevant to the triad sequences as follows; left peak to BD-TP-BD, middle peak to BD-TP-PTMG and PTMG-TP-BD, right peak to PTMG-TP-PTMG.

Above peak assignment for phenyl and carbonyl regions could be confirmed by manifest heteronuclear long range couplings in $2 \mathrm{D}$ HMBC spectra (Figures $3 \mathrm{a}$ and b). In Figure $3 a$, we presented 2D spectrum representing long range coupling between phenyl protons and quaternary carbons. In that spectrum, four cross peaks are shown, which means there are four different heteronuclear long range couplings between phenyl protons and quaternary carbons. In that $2 \mathrm{D}$ spectrum, we can see that middle peaks of quaternary carbon were divided into two cross peaks by two outer peaks of the phenyl proton and the middle peak of phenyl proton has also two cross peaks by two side peaks of quaternary carbons. Another 2D HMBC (Figure 3b) spectrum shows the long range coupling between phenyl proton and carbonyl carbon. Clearly, we can see each line of three peaks of carbonyl carbons corresponds to each single line of phenyl protons, which means that the respective three lines of protons and carbons are originated from same microstructures of the polymer. Of course, these results completely coincide with above peak assignment inferred from integration values of the peaks.

Besides above three groups of NMR peaks in phenyl and carbonyl regions, we could find that the aliphatic regions of ${ }^{13} \mathrm{C}$ NMR spectra also gave us useful information associated with triad sequences of the polymer. We focused the small peaks beside main ones in the ${ }^{13} \mathrm{C}$ NMR spectrum. First of all, for the purpose of exact assignment of the ${ }^{13} \mathrm{C}$ spectra, we must carry out ${ }^{1} \mathrm{H}$ NMR peak assignments for small peaks in aliphatic regions overlapped into the main peaks. It was carried out through the analysis of chemical shift, coupling patterns and ${ }^{1} \mathrm{H}-{ }^{1} \mathrm{H}$ COSY (Figure $3 \mathrm{c}$ ) for those proton peaks. Consequently, the small peaks were found to be related to the terminal tetramethylene group of PTMG that is directly attached to the terephthalic acid unit ( $\mathrm{a}-\mathrm{d}$ in Figure 1). On the basis of the assignment of ${ }^{1} \mathrm{H}$ peaks, the small peaks in aliphatic region of ${ }^{13} \mathrm{C}$ NMR spectra could be easily assigned through the analysis of ${ }^{1} \mathrm{H}-{ }^{13} \mathrm{C}$ COSY spectra (Figure $3 \mathrm{~d}$ and Table II). For example, the small peaks at 65.62 and $25.00 \mathrm{ppm}$ were assigned to be from terminal tetramethylene group of PTMG that have direct linkage to TP unit in the polymer (a, b in Figure 1). Of course, the peaks at 65.33 and $24.88 \mathrm{ppm}$ are from the tetramethylene group of BD portions of PBT/PTMG (A, B in Figure 1). Then, previous two peaks can be considered to contain the information about two triads; PTMG-TP-PTMG and PTMG-TP-BD (PTMG side). And the next two peaks are considered to have information about other two triads; BD-TP-BD and BD-TP-PTMG (BD side). Actually, it is convenient to think that the two sets of two peaks $(65.62,65.33 \mathrm{ppm}$ and $25.00,24.88 \mathrm{ppm})$ respectively contain the information relevant to all four possible triads as different kinds of nuclei. The assignments for each of the lines of the split peak for above five kinds of nuclei along triad sequences are summarized in the Figure 2 and Table II. In short, the major result is that the peaks from five kinds of nuclei in the Table II have respectively some information about the triad sequence of the polymers.

\section{Extraction of Probabilities of Triads}

As previously described, each NMR peak corresponding to the five kinds of nuclei that were assigned in previous section has manifestly different aspects in the point of view of triad sequences. Therefore, it is very promising to use all these peaks to get the information associated with the probabilities of four possible triad 

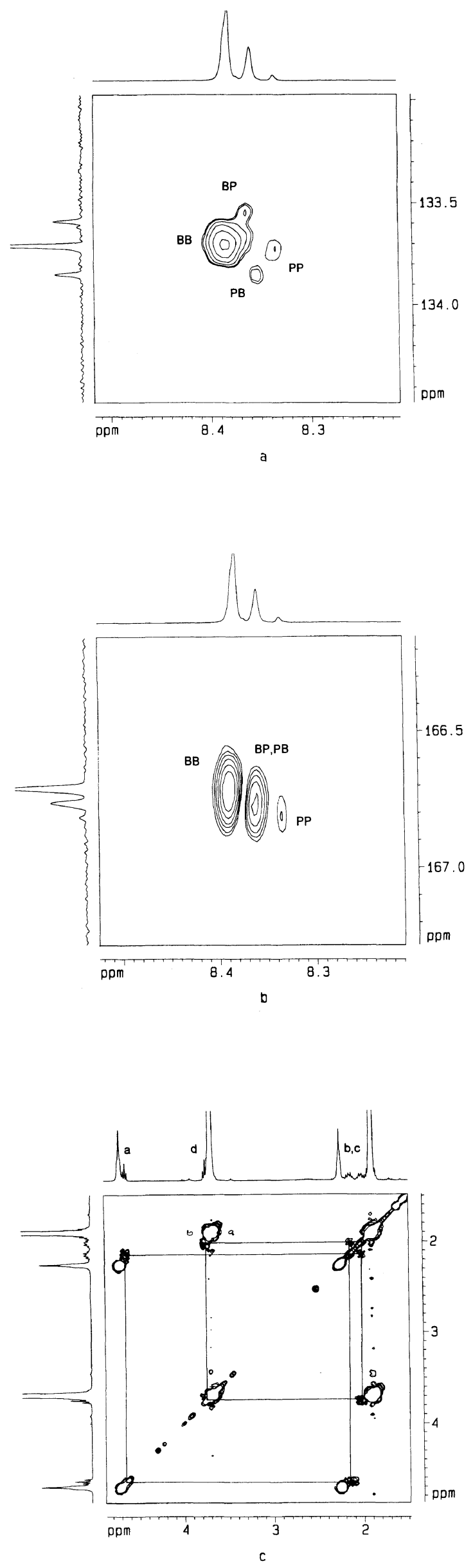

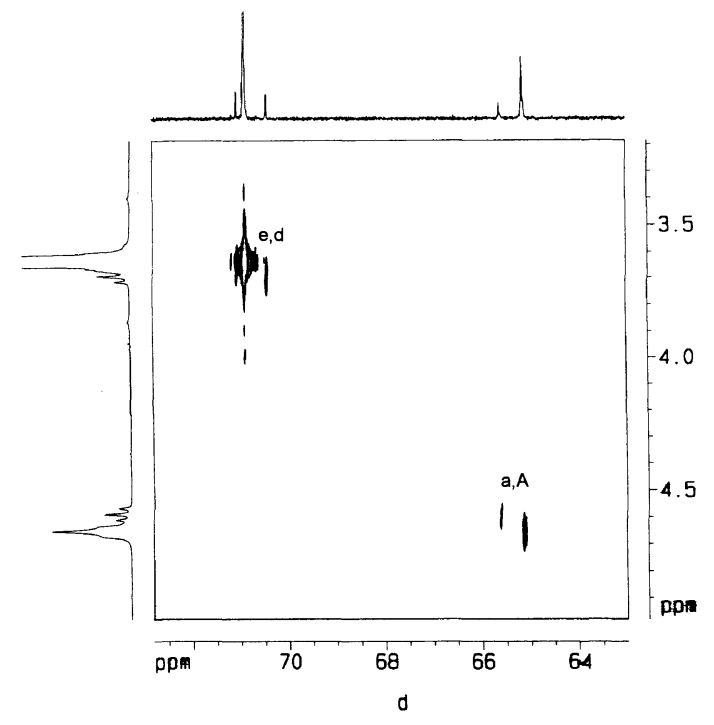

Figure 3. Selected regions of various $2 \mathrm{D}$ NMR spectra of PBT/PTMG (a, HMBC for phenyl proton and phenyl quaternary carbon; b, HMBC for phenyl proton and carbonyl carbon; $c,{ }^{1} \mathrm{H}-{ }^{1} \mathrm{H}$ COSY; $d,{ }^{1} \mathrm{H}^{13}{ }^{13} \mathrm{C}$ COSY spectra of aliphatic proton and carbons).

sequences. Of course, that method is more accurate than those of other authors ${ }^{8-10}$ because we can use simultaneously five independent sets of experimental data. In practice, simple FORTRAN 77 program that performs least square fitting through simplex algorithm was prepared and used to fit experimental data on personal computer. In first, the probabilities of four triads sequences were assumed and the intensities of NMR peaks were calculated, then the intensities of NMR peaks from experiments and calculations were compared. Simplex algorithm (in Numerical Recipe) automatically changed the values for probabilities of triads to minimize the sum of square of error ratio; $\Delta^{2}=\sum[S(\exp )-S(\text { cal })]^{2} /$ $S(\exp )^{2}$. The fitting was continued when the error ratio did not vary at constant level. For each sample, as 5 independent experiments had been performed, ten repeated calculations were carried out for all experiments. For each calculation, the calculated sum of square of error ratio was lower than $2 \times 10^{-4}$. The representative example of experimental and calculated values for the NMR peaks were compared in Table III for sample 1.

\section{Calculation of Parameters Representing the Micro- structures of PBT/PTMG}

The main purpose of this study is the comparison of the microstructures of PBT/PTMG that have been prepared from different polymerization methods. This may be proceeded mainly by making use of interpretation of above probabilities of triads sequences. In addition to those ones, there are other parameters relevant to them giving detailed microstructural information of the polymers. First, from the probabilities of triad sequences, average sequence lengths of hard and soft segments can be calculated as follows; hard segment length, $\mathrm{HSL}=([\mathrm{BB}]+[\mathrm{BP}]) /[\mathrm{BP}]$; soft segment length, $\mathrm{SSL}=([\mathrm{PB}]+[\mathrm{PP}]) /[\mathrm{PB}]$ where $[\mathrm{AB}]$ is the probability of $\mathrm{AB}$ sequence. Because [BP] must be the same as [PB] assuming infinite chain, we used average values for these values in real calculation for simplicity. Calculated 
Table II. Peak assignment of ${ }^{1} \mathrm{H}$ and ${ }^{13} \mathrm{C}$ NMR spectra (from phenol- $d_{6} / \mathrm{TCE}$ solvent) and correspondence between intensities of split NMR lines and linear combinations of probabilities of triad sequences

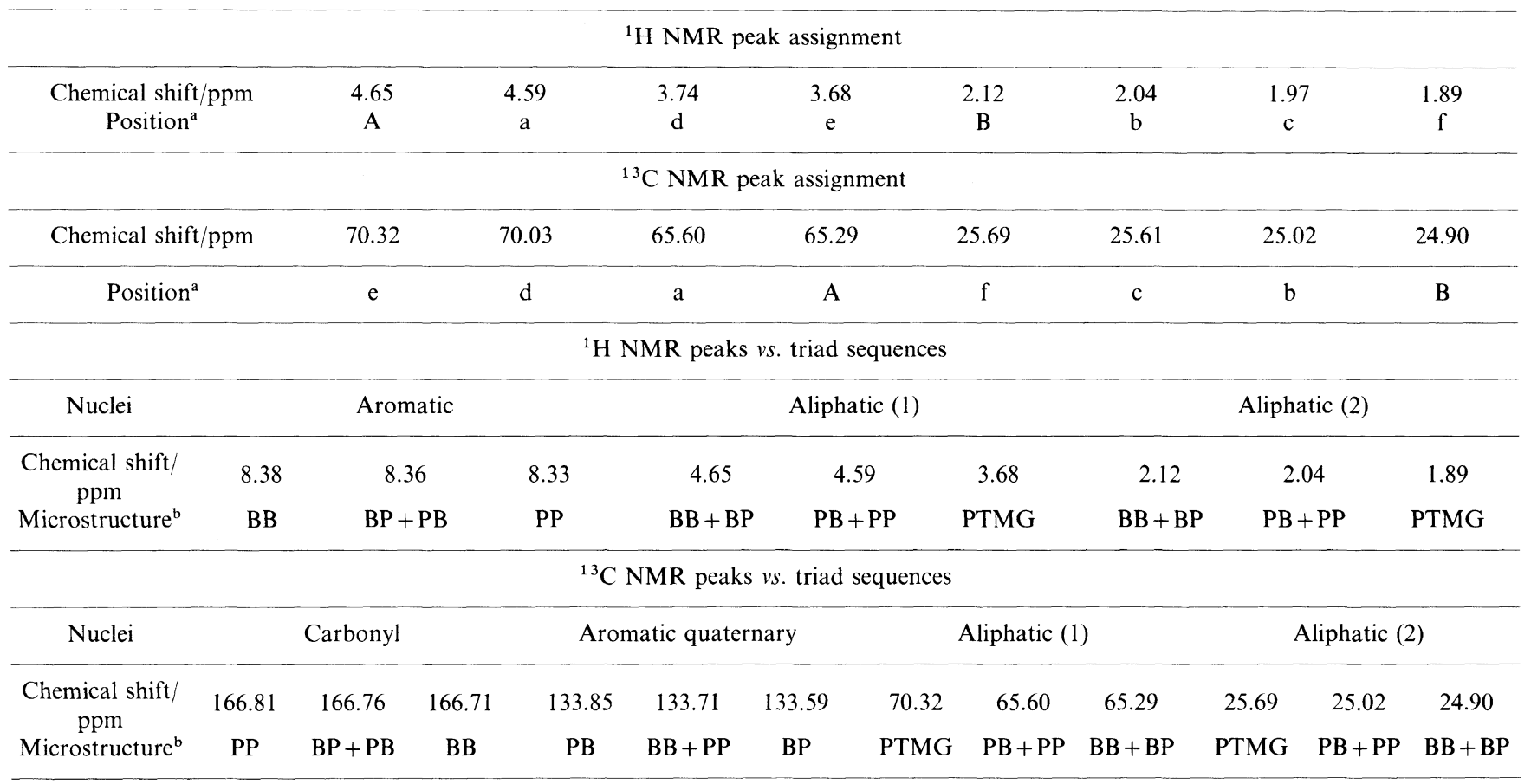

${ }^{\mathrm{a}}$ According to the characters denoted in Figure 1. ${ }^{\mathrm{b}}$ Abbreviations for sequences: BB, BD-TP-BD; BP, BD-TP-PTMG; PB, PTMG-TP-BD; PP, PTMG-TP-PTMG.

Table III. Comparison of experimental and calculated NMR peak intensities of sample 1 (in phenol- $d_{6} / \mathrm{TCE}$ )

\begin{tabular}{|c|c|c|c|c|c|c|c|c|}
\hline \multicolumn{9}{|c|}{ Aromatic proton and carbonyl carbon peaks } \\
\hline Nuclei & \multicolumn{4}{|c|}{ Aromatic ${ }^{1} \mathrm{H}$} & & \multicolumn{3}{|c|}{ Carbonyl ${ }^{13} \mathrm{C}$} \\
\hline Peaks ${ }^{a}$ & BB & $\mathrm{BP}+\mathrm{PB}$ & & PP & & PP & $\mathrm{BP}+\mathrm{PB}$ & BB \\
\hline Exptl & 68.47 & 28.26 & & 3.27 & & 2.87 & 28.18 & 68.95 \\
\hline Calcd & 68.74 & 28.65 & & 2.79 & & 2.79 & 28.65 & 68.74 \\
\hline \multicolumn{9}{|c|}{ Aromatic and aliphatic carbon peaks } \\
\hline Nuclei & \multicolumn{3}{|c|}{ Aromatic quaternary ${ }^{13} \mathrm{C}$} & & \multicolumn{2}{|c|}{ Aliphatic ${ }^{13} \mathrm{C}(1)$} & \multicolumn{2}{|c|}{ Aliphatic ${ }^{13} \mathrm{C}(2)$} \\
\hline Peaks ${ }^{a}$ & PB & $\mathrm{BB}+\mathrm{PP}$ & BP & & $\mathrm{PB}+\mathrm{PP}$ & $\mathrm{BB}+\mathrm{BP}$ & $\mathrm{PB}+\mathrm{PP}$ & $\mathrm{BB}+\mathrm{BP}$ \\
\hline Exptl & 14.70 & 71.07 & 14.23 & & 16.57 & 83.43 & 17.02 & 82.98 \\
\hline Calcd & 14.54 & 71.53 & 14.12 & & 17.32 & 82.86 & 17.32 & 82.86 \\
\hline
\end{tabular}

${ }^{\text {a }}$ Corresponding to the peaks in Table II.

probabilities of triad sequences also can be used to calculate the molecular weight of PTMG in block copolymer. The calculation of molecular weight of PTMG can be performed by the comparison of the integration values of NMR peaks of terminal ( $a$ or $b$ in Figure 1) with those of central part (e or $f$ in Figure 1) of tetramethylene group of PTMG chain. Because direct comparison of small and large peaks can give significant errors in obtaining the ratio of the intensities, we used calculated values for terminal peaks $(P P+P B)$ from above fitting procedure instead of direct integration values of $a$ or $b$. Then, the number average molecular weight can be obtained from that ratio added by appropriate value corresponding to terminal tetramethylene groups and multiplied by 72.12 . In this study, this method was found to be good to get the actual molecular weight of PTMG in the block copolymer. When we consider that the actual soft segment is the sum of PTMG and TP, molecular weight of unit soft segment is M.W. of PTMG +148.12 in block copolymer. Molecular weight of unit hard segment is calculated to be $72.12+148.12=220.24$ for all samples. Conceptually, the product of segment length and molecular weight of unit segment produces the average molecular weight of each segmented block. For example, average molecular weight of soft segmented block is the product of SSL by M.W. of PTMG added by 148.12 and so on. Finally, under the assumption of infinite chain, hard and soft segment contents in block copolymer are easily calculated from such average molecular weights of each segment. In the point of view of chemical composition, because the chain can be considered schematically consisting of alternative hard and soft segments, the HSC, for example, can be calculated to be the average molecular weight of hard segment divided by the sum of average molecular weight of hard and soft segment. All above calculated 
B. Min and E. BANG

Table IV. Comparison of calculated parameters relevant to the microstructures of two samples (in phenol- $d_{6} / \mathrm{TCE}$ )

\begin{tabular}{|c|c|c|c|c|c|c|c|c|c|c|}
\hline \multirow{3}{*}{ Samples } & \multicolumn{10}{|c|}{ Calculated parameters ${ }^{a}$} \\
\hline & \multicolumn{4}{|c|}{ Probabilities of triad sequences } & \multicolumn{2}{|c|}{ Sequence length ${ }^{b}$} & \multirow{2}{*}{$\begin{array}{l}M_{n}^{\mathrm{c}} \text { of } \\
\text { PTMG }\end{array}$} & \multicolumn{2}{|c|}{$\begin{array}{l}\text { Average molecular } \\
\text { weight of segment }\end{array}$} & \multirow{2}{*}{$\begin{array}{c}\text { HSC } \\
\%\end{array}$} \\
\hline & BB & $\mathrm{BP}$ & $\mathrm{PB}$ & PP & HSL & SSL & & $\mathrm{HMW}^{\mathrm{d}}$ & $\mathrm{SMW}^{\mathrm{e}}$ & \\
\hline \multirow[t]{2}{*}{1} & 0.684 & 0.142 & 0.143 & 0.031 & 5.787 & 1.218 & 1945.2 & 1274.7 & 2549.8 & 33.34 \\
\hline & $(0.002)$ & $(0.001)$ & $(0.001)$ & $(0.001)$ & $(0.027)$ & $(0.008)$ & $(23.11)$ & $(6.08)$ & (34.94) & $(0.27)$ \\
\hline \multirow[t]{2}{*}{2} & 0.683 & 0.145 & 0.144 & 0.028 & 5.734 & 1.197 & 1967.3 & 1262.9 & 2534.6 & 33.30 \\
\hline & $(0.002)$ & $(0.002)$ & $(0.001)$ & $(0.003)$ & $(0.047)$ & $(0.020)$ & $(21.70)$ & $(10.31)$ & $(52.83)$ & $(0.31)$ \\
\hline$\left|t_{0}\right|^{\mathbb{g}}$ & 0.165 & 0.761 & 1.192 & 0.759 & 0.981 & 0.738 & 0.694 & 0.981 & 0.188 & 0.052 \\
\hline
\end{tabular}

${ }^{a}$ For each samples, the set of parameters were calculated for 5 independent experiments and average values and standard error of the mean (in parenthesis) were presented. ${ }^{\mathrm{b}} \mathrm{HSL}$, sequence length of hard segment; SSL, sequence length of soft segment. ${ }^{\mathrm{c}}$ Number average molecular weight of PTMG calculated from intensities of NMR spectra. ${ }^{d}$ Number average molecular weight of hard segment calculated from intensities of NMR spectra. ${ }^{\mathrm{e}}$ Number average molecular weight of soft segment calculated from intensities of NMR spectra. ${ }^{\mathrm{f}} \mathrm{Calculated}$ hard segment contents. ${ }^{\mathrm{g}}$ Test statistics: $t_{0}=(\langle X\rangle-\langle Y\rangle) / s_{\mathrm{p}}\left(1 / n_{1}+1 / n_{2}\right)^{1 / 2}$ where $s_{\mathrm{p}}{ }^{2}=\left(\sum_{i}\left(X_{i}-\langle X\rangle\right)^{2}+\sum_{i}\left(Y_{i}-\langle Y\rangle\right)^{2}\right) /\left(n_{1}+n_{2}-2\right), n_{1}$ and $n_{2}$ are number of experiments for each sample.

parameters are given in Table IV in terms of averages of five experiments and standard error of the mean.

\section{Comparison of Microstructures of PBT-PTMG According to the Polymerization Methods}

When we compare the calculated parameters for two samples in Table IV we can find that both samples exhibit $\mathrm{BD}-\mathrm{TP}-\mathrm{BD}$ sequences as main sequence structure of the chain. One can expect that there are few frequencies of two or more sequential PTMGs considering the molecular weight of PTMG. Assuming there are no probabilities of PTMG-TP-PTMG sequences and the length of the polymer is infinite, the theoretical probabilities of BD-TP-BD and BD-TP-PTMG (BD or PTMG site) can be calculated to be 0.657 and 0.172 for the copolymer having HSC of $33.3 \%$ when the molecular weight of PTMG in the copolymer is equal to $1982(2000-18)$. From the table, we can easily find that the probabilities of the BD-TP-BD have larger values compared to that from previous assumptions. The probabilities of BD-TP-PTMG (BD site) and PTMG-TP-BD (PTMG site) have smaller values. Moreover, the probabilities of PTMG-TP-PTMG are found to be about 3\% for both samples. From above calculated probabilities, we can see that there were formations of some PTMG-TPPTMG sequences and it made the polymer slightly blockier than expected from above simple calculation. Actually, when we assume the Bernoullian statistics with which the parameters controlling the chain propagation in polymerization is hard or soft segment contents, the theoretical probabilities of above triads sequences are 0.686 (BD-TP-BD), 0.142 (BD-TP-PTMG, and PTMG-TP-BD) and 0.030 (PTMG-TP-PTMG), respectively. For sample 1, the calculated probabilities are very close to the theoretical ones. However, for sample 2, we can find the tendency that the average values corresponding to BD-TP-BD and PTMG-TPPTMG are lower than the theoretical values whereas those corresponding to the BD-TP-PTMG and PTMGTP-BD are higher than theoretical ones. If it is true, this means that sample 2 is less blocky than sample 1 due to discrepancy from Bernoullian statistics. Comparing average sequence length calculated from the probabilities of triad sequences enforce above reasoning.
Before going to the serious comparison of the data of two samples, it is worth considering general features of the sequence lengths. From the Table IV, we can see that the calculated (from measurements) average hard segment sequence lengths are in the range of $5.70-5.80$ and soft segment lengths are about 1.20 for two samples. As for the soft segment length, these values correspond to the fact that among ten soft segments, about two have two adjacent PTMG while about eight have unit PTMG segment. Actually, when we designed the polymerization of sample 2 we expected that there were low frequencies of the PTMG-TP-PTMG sequences due to relatively short reaction time of PTMG molecules. The experimental data show that the sequence lengths of soft segment are still about 1.2 that is similar to that of sample 1 though slight decreasing of sequence length in sample 2 could be detected.

At this point, we intend to mention about the suitability of the NMR methods to discriminate above minute differences of the microstructures of the copolymers. Though many workers mentioned about the wealthy information relevant to the NMR method to elucidate microstructures of the polymer, ${ }^{8-10}$ they also pointed out the limitation of the method to undergo exact quantification. We think the limitations are mainly attributed to the overlap of the peaks in the case of proton NMR and poor signal to noise ratio $(\mathrm{S} / \mathrm{N}$ ratio) in the case of carbon NMR. In this study, as previously described, we found that aromatic region of proton NMR could give well-resolved lines under phenol/TCE solvent and the simultaneous fitting of several parts of carbon signals overcome the integration errors due to low $\mathrm{S} / \mathrm{N}$ ratio in ${ }^{13} \mathrm{C}$ NMR. Therefore we think that the errors in measurement have been greatly reduced compared to previous studies. ${ }^{8-10}$ This can be verified by very low level of standard error of the mean (below $1 \%$ for most cases) of extracted parameters for 5 independent experiments (Table IV). For two samples, to know whether there were real meaningful differences in extracted parameters or not, statistical tests were performed. According to statistics, ${ }^{16}$ if one intends to compare the properties of two samples from two differently designed experiments, one must perform several experiments and calculate test statistic for two sam- 
An NMR Study of Sequence Distribution of PBT/PTMG

Table V. Calculated parameters relevant to the microstructures of two samples in $\mathrm{CDCl}_{3}$

\begin{tabular}{|c|c|c|c|c|c|c|c|c|c|c|}
\hline \multirow{3}{*}{ Samples } & \multicolumn{10}{|c|}{ Calculated parameters $^{\mathrm{a}}$} \\
\hline & \multicolumn{4}{|c|}{ Probabilities of triad sequences } & \multicolumn{2}{|c|}{ Sequence length ${ }^{\mathrm{b}}$} & \multirow{2}{*}{$\begin{array}{l}M_{n}^{\mathrm{c}} \text { of } \\
\text { PTMG }\end{array}$} & \multicolumn{2}{|c|}{$\begin{array}{l}\text { Average molecular } \\
\text { weight of segment }\end{array}$} & \multirow{2}{*}{$\frac{\text { HSC }^{f}}{\%}$} \\
\hline & BB & $\mathrm{BP}$ & $\mathrm{PB}$ & $\mathrm{PP}$ & HSL & SSL & & $\mathrm{HMW}^{\mathrm{d}}$ & $\mathrm{SMW}^{\mathrm{e}}$ & \\
\hline 1 & 0.685 & 0.142 & 0.144 & 0.03 & 5.79 & 1.22 & 1981 & 1275 & 2598 & 32.92 \\
\hline 2 & 0.678 & 0.145 & 0.148 & 0.029 & 5.62 & 1.21 & 1985 & 1238 & 2581 & 32.42 \\
\hline
\end{tabular}

${ }^{a}$ For these solutions detailed error analysis were not performed and only one set of typical experimental results have been presented. ${ }^{b}$ HSL, sequence length of hard segment; SSL, sequence length of soft segment. ${ }^{\mathrm{c}}$ Number average molecular weight of PTMG calculated from intensities of NMR spectra. ${ }^{\mathrm{d}}$ Number average molecular weight of hard segment calculated from intensities of NMR spectra. ${ }^{\mathrm{e}}$ Number average molecular weight of soft segment calculated from intensities of NMR spectra. ${ }^{\mathrm{f}}$ Calculated hard segment contents.

ples. Then, test statistic is compared with the critical value at intended significance level. If the test statistic is less than the critical value, one thinks the two samples have no significant difference and vice versa. In the comparison, the critical value depends on number of experiments and intended accuracy of the comparison. For example, if one compare two samples with $95 \%$ certainty the critical value is 2.776 when the numbers of experiments are $5 .^{16}$ If we compare the two samples with $60 \%$ certainty that is 0.941 . In the bottom of Table IV we presented test statistic for each parameter. At the glance of the calculated parameters, we can see that most of calculated test statistics have lower values compared to above critical values. If we hypothesis the calculated parameters are different between two samples, any of the parameters is not found to belong to the acceptance region of $95 \%$ certainty. Some parameters go into or are close to that of $60 \%$ certainty, especially the lengths of segments. This means that we can say that there have some differences in the microstructures of two samples relevant to those parameters with $60 \%$ certainty. However, through above statistical analysis, we conclude that the effect of previous two polymerization methods is too small to be detected in spite of using these relatively accurate analytical method.

For both polymers, calculated molecular weights of PTMG are very close to that of starting PTMG. Actually, the molecular weight of PTMG in the block copolymer must have the values less than that of original. So to say, because one molecule of water must be removed for PTMG to be polymerized, the upper limit of the molecular weight of the PTMG is 1982 if the moelcular weight of original PTMG is exactly 2000 . From the table, we can find that the calculated molecular weights of PTMGs of two samples are not significantly different from 1982. If the values are significantly different, it can be suspected that the degradation of PTMG chain may occur when the experimental error and inaccuracy of the maker's specification are not so large. In line with these aspects, it seems that there are not so much degradation of PTMG in both of the samples in contrast to other reports. ${ }^{14,17}$ Because there are not so significant differences in sequence lengths and M.W. of PTMG, average molecular weight of each segmented block must be also similar. In the two samples, it is interesting that the average molecular weight of soft segments has the value that is nearly twice as much as that of hard segment. This has been directly reflected in the calculation of contents of each segments. We can see from the table that the calculated HSC of both samples are nearly identical to the fed contents $(33.3 \%)$. The fact that the HSC calculated from only experimental data under the minimum assumption is very close to the fed ratio indicate that our method is very accurate. Another fact we want to point out is spectroscopic evidence of completeness of the polymerization reaction. All above calculations (from measurement), in principle, based on the information are associated with chemical bonds between starting materials. Therefore the consistency of the calculated (measured) and fed chemical compositions means the polymerization went nearly complete.

All above results are from the solutions using phenol$d_{6} /$ TCE. Besides phenol- $d_{6} /$ TCE, we tried to perform NMR measurement for the solution of $\mathrm{CDCl}_{3}$. In general, the PBT/PTMG of low contents of hard segment can be dissolved in $\mathrm{CDCl}_{3}$ apparently. Higashiyama et $a{ }^{8}{ }^{8}$ have undergone NMR study of sequence distribution of PBT/PTMGs containing relatively wide range of HSC $(20-40 \%)$ using $\mathrm{CDCl}_{3}$. However, in this study, we found that all ingredients of the polymer cannot be fully dissolved into the $\mathrm{CDCl}_{3}$. In Table $\mathrm{V}$, we presented the calculated parameters of two samples in $\mathrm{CDCl}_{3}$ solutions. Through the quick comparison of the results with those of previous ones (from phenol- $d_{6} / \mathrm{TCE}$ ), one can find rather difference between them. Among several parameters, the calculated hard segments contents for two samples shows conclusive results in comparison between two solvents. When we see the level of differences and previous standard deviation of HSC's we think there are manifest difference between two solvents. Quantitatively, the fact that calculated HSC is less than $33 \%$ means that some parts of hard segments of two samples (samples 1 and 2) were not fully dissolved in $\mathrm{CDCl}_{3}$. Though we will not present the detailed experimental results, we found that when HSC was increasing above $33.3 \%$ the non-dissolving part was more rapidly increasing. From the Table $\mathrm{V}$ we can see that the probability of PTMG-TP-PTMG sequence and soft segment length have larger values than those of phenol$d_{6} /$ TCE solutions. However, we think it is hard to find any useful information relevant to the microstructure of the copolymer from this $\mathrm{CDCl}_{3}$ solution because the solution cannot reveal the whole properties of the polymers.

Besides HSC, there is another thing worth to say when we closely examine NMR spectra of $\mathrm{CDCl}_{3}$ solution. 

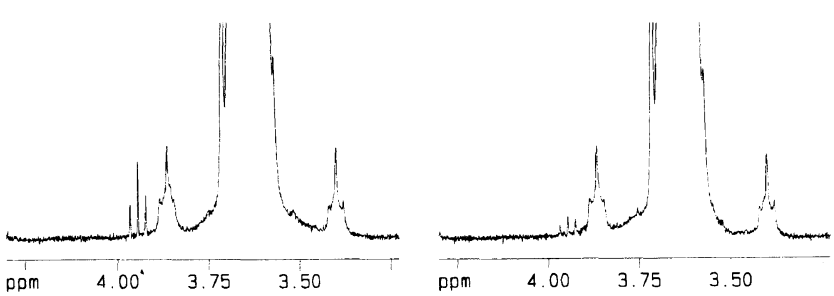

Figure 4. Comparison of selected aliphatic regions of ${ }^{1} \mathrm{H}$ NMR spectra of two samples (samples 1 and 2) showing the terminal protons of PTMG which exist in the end of the whole molecule of PBT/PTMG (in $\mathrm{CDCl}_{3}$ ).

Figure 4 shows the comparison of interested aliphatic region of magnified ${ }^{1} \mathrm{H}$ spectra of two samples. In the figure, the sharp and manifest triplets can be assigned to the peaks due to the protons of PTMG's adjacent to terminal $-\mathrm{OH}$. It is very interesting to see the difference of the intensities of the signals. Under the assumption of non-existence of free PTMG, the large NMR signal for the terminal group means there are many copolymer molecules that have the PTMG as terminal group. Considering that the number average molecular weights of two samples are comparable, above experimental results denote that sample 1 has more molecules that have terminal PTMG compared to sample 2. If we go back to the Table IV we can get consistent result from the comparison of calculated values for the probabilities of BD-TP-PTMG and PTMG-TP-BD. In infinite chain of block copolymer, it is a matter of course that the probabilities of BD-TP-PTMG and PTMG-TP-BD are the same. In real polymers that have finite length of the chain, if the numbers of hard and soft segmented blocks are equal, the probabilities of two sequences must be also equal. In contrast, unequal probability of above two sequences means unequal number of hard and soft segment block. In alternation of schematic sequence of the copolymers, inequality of the two kinds of sequences must only be attributed by status of the terminal. Therefore the fact that the relative higher value for the probabilities of PTMG-TP-BD for sample 1 means that there are more molecules that have soft segment or PTMG as terminal. For sample 2, the result was reversed and it means that it is difficult to find the PTMG in terminal. This fact well agrees to the previous results from ${ }^{1} \mathrm{H}$ NMR using $\mathrm{CDCl}_{3}$ though the solvents and the chemical composition of the solution are different in two cases.

Up to now, we presented the results of calculations of various parameters relevant to the microstructures of PBT/PTMG. For two polymers made from different polymerization methods, we discussed fully the differ- ences of the parameters and physicochemical natures. In regarding our main object, we could not obtain dramatic results in this study except it were shown that the microstructure of two samples could be closely evaluated by modified NMR methods. In other words, there were no significant difference in microstructures between two samples. The main reason of the results may be considered that there are sufficiently long reaction time and all the units in the copolymer were randomized during this time. The fact that the calculated probabilities of the four triads sequence well accords to the Bernoullian statistics reinforce above reasoning. The relatively long chain of PTMG is also considered to prevent the structure of the samples from significant difference. To have great difference in the microstructure, it is necessary for the polymer to be much varied in blockiness owing to the conditions of preparation. However, for our samples, in view of above results, unit soft segment is considered too long to form the chemical bonds for formation of adjacent two or more molecules. From those point of view, under the condition of short reaction time and using low molecular weights of PTMG, controlling of microstructures and their measurement are valuable if it has some relationship with physicochemical properties of the polymers.

\section{REFERENCES}

1. R. J. Cella, J. Polym. Sci., Polym. Symp., 42, 727 (1973).

2. G. Holden, Encycl. Polym. Sci. Eng., 5, 416 (1986).

3. A. Schmidt, W. S. Veeman, V. M. Litvinov, and W. Gabrielse, Macromolecules, 31, 1652 (1998).

4. L.-L. Zhu and G. Wegner, Makromol. Chem., 182, 3625 (1981).

5. G. Wegner, T. Fujii, W. Meyer, and G. Lieser, Dye. Angew. Makromol. Chem., 74, 295 (1978).

6. L.-L. Zhu, G. Wegner, and U. Bandara, Makromol. Chem., 182, 3639 (1981).

7. R. M. Briber and E. L. Thomas, Polymer, 26, 8 (1985).

8. A. Higashiyama, Y. Yamamoto, R. Chûjô, and M. Wu, Polym. J., 24, 1345 (1992).

9. B. Jacques, J. Devaux, R. Legras, and E. Nield, J. Polym. Sci., Polym. Chem. Ed., 34, 1189 (1996)

10. R. A. Newmark, J. Polym. Sci., Polym. Chem. Ed., 18, 559 (1980).

11. W. R. Sorenson and T. W. Campbell, "Preparative Methods of Polymer Chemistry," Interscience, New York, N.Y., 1951.

12. C. M. Boussias, R. H. Peters, and R. H. Still, J. Appl. Polym. Sci., 25, 855 (1980).

13. D. H. Baik, M. S. Lee, B. Y. Jeon, and M. S. Han, J. Kor. Fiber. Soc., 31, 613 (1994).

14. S. Fakirov and T. Gogeva, Makromol. Chem., 191, 603 (1990).

15. S. Fakirov and T. Gogeva, Makromol. Chem., 191, 615 (1990).

16. J. C. Miller and J. N. Miller, "Statistics for Analytical Chemistry," Ellis Horwood Ltd., Chichester, 1988.

17. A. Davis and J. H. Golden, J. Am. Chem. Soc., Div. Polym. Chem. Prepr., 5/20, 461 (1964). 